\title{
Fisioter apia em mastologia oncológica: rotinas do Hospital do Câncer III / INCA
}

\author{
Physical Therapy in Breast Cancer: clinical protocol at the Cancer Hospital III / I NCA
}

\author{
Anke Bergmannn ${ }^{1}$, Maria J ustina Padula Ribeiro², Elisangela Pedrosa ${ }^{3}$, Erica Alves Nogueira4a ${ }^{4}$ Ana Cristina Gonçalo Oliveira ${ }^{5}$
}

\begin{abstract}
Resumo
A pós o tratamento do câncer da mama, várias complicações vêm sendo relatadas na literatura. A fisioterapia desempenha um importante papel na prevenção, minimização e tratamento dos efeitos adversos do tratamento do câncer da mama. A implantação da rotina de atendimento fisioterapêutico para pacientes submetidas a tratamento para câncer da mama tem como objetivo principal a prevenção de complicações através de condutas e orientações domiciliares, e o diagnóstico e intervenção precoce, visando melhorar a qualidade de vida e a redução dos custos pessoais e hospitalares. Além disso, possibilita a formação de um banco de dados contendo informações sobre fatores de risco e incidência de complicações, avaliação de condutas empregadas e o planejamento do serviço. As condutas aqui apresentadas sofrem constantes modificações de acordo com o incremento de técnicas fisioterapêuticas, disponibilidade de recursos materiais e humanos. A nossa proposta é divulgar o trabalho realizado, para que possa ser conhecido e discutido entre os outros profissionais e serviços de fisioterapia oncológica.
\end{abstract}

Palavras-chave: N eoplasia mamária; Fisioterapia; Reabilitação; Complicações.

\begin{abstract}
Various complications following breast cancer treatment have been reported in the literature. Physical therapy plays an important role in prevention, reduction, and treatment of adverse effects from breast cancer treatment. The implementation of routine physical therapy for patients undergoing breast cancer treatment aims to prevent complications through homecare measures and orientation as well as early diagnosis and intervention, seeking to improve quality of life and reduce personal and hospital costs. It also allows the formation of a database on risk factors and incidence of complications, evaluation of various approaches, and service plans. The approaches shown here undergo constant changes aimed at improving physical therapy techniques and availability of human and material resources. $\mathrm{O}$ ur aim is to publicize the work accomplished to date for its discussion by other professionals as well as by physiotherapy services in other cancer centers.
\end{abstract}

Key words Breast cancer; Physical therapy; Rehabilitation; Complications.

\footnotetext{
${ }_{1}^{1}$ Fisioterapeuta, D outora em Ciências da Saúde - EN SP/FIO CRUZ

2 Fisioterapeuta, Especialista em Fisioterapia em Cancerologia - SBFC

${ }^{3}$ Fisioterapeuta, Especializada em Fisioterapia em Oncologia - IN CA

${ }^{4}$ Fisioterapeuta, Especializada em Fisioterapia em Oncologia - IN CA

${ }^{5}$ Fisioterapeuta, Especializada em Fisioterapia em Oncologia - IN CA

Trabalho realizado no Serviço de Fisioterapia do H ospital do Câncer III / Instituto N acional de Câncer

Endereço para correspondência: Anke Bergmann - Rua Visconde de Santa Izabel, 274. Serviço de Fisioterapia / H CIII / IN CA. E-mail: abergmann@inca.gov.br - fisio.hc3@inca.gov.br
} 


\section{INTRO DUÇÃO}

Em decorrência do tratamento do câncer da mama, várias complicações têm sido relatadas na literatura. As complicações cirúrgicas ocorrem, com diferenças de intensidade e incidência, tanto nas técnicas conservadoras como nas radicais. 0 tratamento adjuvante, seja ele radioterápico, quimioterápico ou hormonioterápico, se sobrepõe às seqüelas cirúrgicas, aumentando os riscos de complicações ${ }^{1}$.

A fisioterapia, quando iniciada precocemente, desempenha um importante papel na busca da prevenção das complicaçōes advindas do tratamento do câncer da mama, favorecendo o retorno às atividades de vida diária, e melhor qualidade de vida $2,3,4,5$.

0 programa de fisioterapia deve ser realizado em todas as fases do câncer da mama: prétratamento (diagnóstico e avaliação); durante o tratamento (quimioterapia, radioterapia, cirurgia, e hormônioterapia); após o tratamento (período de seguimento); na recorrência da doença e nos cuidados paliativos. Em cada uma dessas fases, é necessário conhecer e identificar as necessidades do paciente, os sintomas e suas causas, e 0 impacto desses nas atividades de vida diária. A conduta fisioterapêutica deve, então, ser traçada através de orientações domiciliares e tratamentos específicos ${ }^{6,7}$.

0 presente artigo tem como objetivo divulgar as condutas empregadas no serviço de fisioterapia do H ospital do Câncer III, unidade responsável pelo atendimento exclusivo de pacientes com câncer da mama do Instituto $\mathrm{N}$ acional de Câncer.
DESCRIÇ̃O DAS ROTINAS DO SERVIÇO DE FISIOTERAPIA

O s atendimentos de rotina são realizados de acordo com a figura 1 .

\section{Pré-operatório}

População alvo: todas as pacientes com indicação cirúrgica de linfadenectomia axilar, biópsia do linfonodo sentinela e reconstrução mamária.

Atendimento: individual

0 bjetivos: estabelecimento do contato fisioterapeuta $x$ paciente; avaliação (anamnese e exame físico); orientações para o pós-operatório imediato; indicação de fisioterapia préoperatória.

Conduta: apresentação do profissional e dos objetivos da fisioterapia junto ao paciente e esclarecimento da conduta cirúrgica indicada; avaliação fisioterapêutica; orientação em relação aos cuidados iniciais com 0 membro superior (M S), exercícios a $90^{\circ}$ com o M S até a retirada dos pontos e retorno gradativo às atividades de vida diária (AVD 's).

\section{Pós-operatório imediato ( $1^{\circ}$ PO)}

População alvo: todas as pacientes submetidas à linfadenectomia axilar, biópsia do linfonodo sentinela e reconstrução mamária.

Atendimento: individual (avaliação) e em grupo (conduta).

O bjetivos: prevenção de complicações e melhora de sintomatologias.

Conduta: avaliação; orientação quanto aos cuidados com o membro superior, exercícios a $90^{\circ}$ com o MS até a

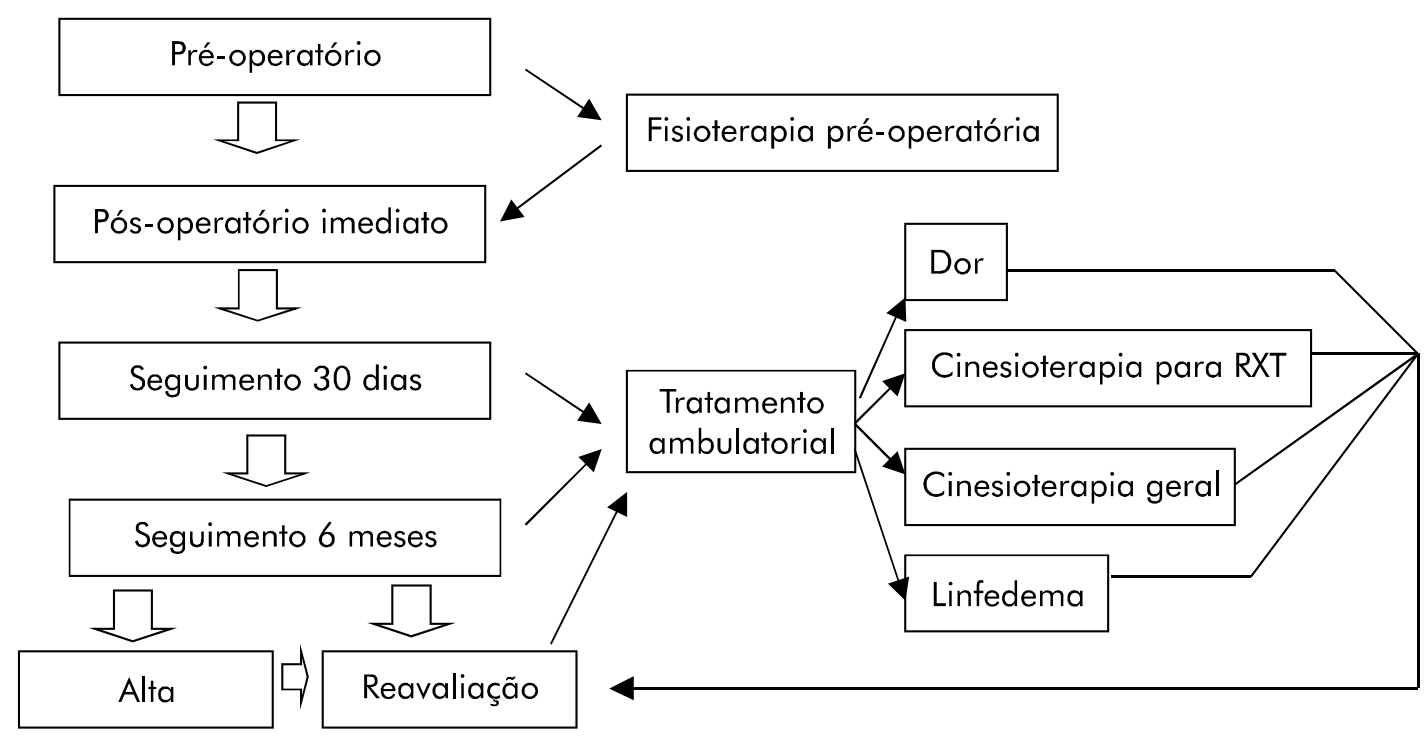

Figura 1. Rotina de Fisioterapia em Mastologia O ncológica / HC III - INCA 
retirada dos pontos, retorno gradativo às AVD's e posicionamento no leito; relaxamento cervical; cinesioterapia ativo-assistida de baixa amplitude com os membros superiores; cinesioterapia respiratória e tratamento sintomatológico.

\section{Consultas de seguimento (trinta dias e seis meses)}

População alvo: pacientes submetidas à linfadenectomia axilar, biópsia do linfonodo sentinela e reconstrução mamária com 30 e 180 dias de pós-operatório.

Atendimento: individual.

O bjetivos: detecção precoce de complicações. Conduta: avaliação fisioterapêutica; orientações específicas segundo sintomatologia apresentada; adaptação de órteses e próteses (quando indicadas); encaminhamento aosgrupos de tratamento, quando necessário.

Atendimento fisioterapêutico ambulatorial para reavaliação de seguimento

Entre os períodos de seguimento, as pacientes que necessitam de atendimentos fisioterapêuticos são orientadas a marcar consulta com a fisioterapia, através do encaminhamento de qualquer profissional de saúde da instituição. É realizada avaliação padronizada e conduta específica segundo a sintomatologia apresentada. Q uando necessário, as pacientes são encaminhadas para os grupos de tratamento específico.

\section{Atendimento fisioterapêutico nas internações clínicas}

D urante a internação clínica, as pacientes são encaminhadas à fisioterapia através de parecer emitido pelos profissionais de saúde. São realizados atendimentos fisioterapêuticos específicos abrangendo todas as clínicas (pneumo-funcional, neurológica, ortopédica, oncológica etc.). Q uando necessário, são adaptadas órteses e próteses visando estabilização segmentar.

Ambulatório para tratamento fisioterapêutico das complicações

\section{Cinesioterapia Geral}

População alvo: pacientes com amplitude incompleta de movimento (AIM).

Atendimento: grupo.

O bjetivos: recuperar a amplitude articular de movimento (ADM) dos membros superiores

Conduta: cinesioterapia ativo-assistida; al ongamento global; relaxamento cervical e orientações específicas.

Consultas semanais: uma

Tempo de atendimento: 1 hora

\section{Cinesioterapia para radioterapia}

População alvo: pacientes com AIM , não apresentando posicionamento adequado para início do tratamento radioterápico.

Atendimento: grupo.

O bjetivos: proporcionar ganho da amplitude articular para posicionamento na radioterapia

C onduta: cinesioterapia ativa-assistida; alongamento global; relaxamento cervical e orientações específicas.

Consultas semanais: duas

Tempo de atendimento: 1 hora

\section{Linfedema}

População alvo: de acordo com o protocolo de tratamento do linfedema.

Atendimento: individual.

O bjetivos: minimizar e controlar o volume do membro. Conduta: auto-massagem linfática; exercícios linfomiocinéticos; enfaixamento compressivo e orientações específicas relacionadas aos cuidados com a pele.

Consultas semanais: duas

Tempo de atendimento: 30 minutos

\section{Dor}

População alvo: pacientes com quadro álgico, sem resposta à orientação domiciliar.

Atendimento: grupo.

O bjetivos: minimização do quadro álgico.

Conduta: cinesioterapia ativo-assistida; alongamento global; relaxamento cervical; auto-massagem linfática; descolamento cicatricial e orientações específicas.

\section{Consultas semanais: uma}

Tempo de atendimento: 1 hora

\section{AVALIAÇÕES FISIOTERAPÊUTICAS}

A avaliação fisioterapêutica deve ser iniciada no pré operatório, objetivando conhecer as alterações pré-existentes e identificar os possivveis fatores de riscos para as complicações pós-operatórias (figura 2) ${ }^{7}$. Q uando necessário, é instituído tratamento fisioterapêutico nessa etapa, visando minimizar e prevenir as possíveis seqüelas identificadas

N o pós-operatório imediato, a avaliação deve buscar identificar alterações neurológicas ocorridas durante 0 ato operatório, presença de sintomatologias ál gicas, edema linfático precoce, e alterações na dinâmica respiratória (figura 3). N o período de seguimento, e durante a terapia adjuvante, a avaliação deverá priorizar a detecção precoce de complicações, sejam elas linfáticas, posturais, funcionais, motoras e/ou respiratórias (figuras 4 e 5). 


\section{CONDUTAS E ORIENTAÇ̃̃ES FISIOTERAPÊUTICAS}

As condutas fisioterapêuticas são realizadas através de orientações domiciliares e tratamentos específicos ambulatoriais e hospitalares, segundo fase de tratamento e sintomatologias apresentadas. Apresentamos um quadro resumido das condutas fisioterapêuticas nas principais complicações do tratamento do câncer da mama (quadro 1) 2,6,8,9 .

\section{Posicionamento e mudanças de decúbito}

0 posicionamento adotado no pós-operatório imediato deverá ser o mais confortável possível; 0 membro superior homolateral à cirurgia pode ser el evado $a+30^{\circ}$ utilizando apoio de travesseiros, não havendo necessidade de mantê-lo em posição de drenagem constante. As mudanças de decúbito são orientadas a serem realizadas em decúbito lateral (DL) oposto. Para as pacientes submetidas à reconstrução com retalho miocutâneo do músculo reto abdominal, o posicionamento adequado é com a cabeceira elevada e semi-flexão de joelhos ${ }^{6}$.

\section{Mobilização do membro superior}

A aderência dos retalhos cutâneos e a cicatrização da ferida operatória são prejudicadas pelas forças tensionais provocadas pelas incursões respiratórias e movimentos do ombro. A contração muscular aumenta a circulação de retorno, fazendo com que a drenagem linfática fisiológica preencha o espaço morto, favorecendo o acúmulo de líquido na cavidade. A imobilização total do $\mathrm{M} S$ favorece a aderência das bordas cirúrgicas, diminuindo a formação do seroma, entretanto leva a retrações importantes, limitando a ADM , aumentando risco de outras complicaçõe $e^{6,8}, 9,10$.

Visando prevenir complicações relacionadas à restrição articular e linfedema, sem aumentar o risco da formação do seroma, a mobilização do braço deve ser iniciada no primeiro dia após a cirurgia, limitada a 90 ำ de flexão, abdução e rotação externa do ombro homolateral à cirurgia, respeitando o limite de tolerância da paciente. A pós a retirada dos pontos e do dreno, não havendo intercorrências proibitivas, orientamos a mobilização completa da amplitude articular ${ }^{6-10}$.

\section{Controle de sintomas álgicos}

As pacientes devem ser encorajadas a iniciar os movimentos ativos de membro superior (limitado a 90 응 até a retirada dos pontos), relaxamento cervical e mobilização escapular desde $01^{\circ}$ pós-operatório. Se houver presença de parestesia em região inervada pelo intercostobraquial, a dessensibilização com diferentes texturas pode ser realizada. Pode-se ainda fazer uso da TENS, corrente interferencial e diadinâmica, crioterapia, e drenagem linfática manual (DLM) para melhora dos sintomas ${ }^{6,11,12}$.

\section{Prevenção e tratamento de alterações posturais}

As pacientes devem ser orientadas a andar com a coluna ereta, cabeça na linha do horizonte, e braços pendentes ao lado do corpo. Para as pacientes submetidas à mastectomia, a prótese mamária de silicone externa deve ser adaptada após a al ta do curativo. Antes, pode ser utilizada prótese de espuma ou material similar (mais leve), o mesmo ocorrendo durante a radioterapia. As mulheres submetidas à cirurgia conservadora podem requerer uso de prótese parcial, a fim de proporcionar simetria mamária. ${ }^{6}$

As diversas técnicas e condutas fisioterapêuticas aplicadas para melhora da postura (RPG, Pilates, antiginástica, cinesioterapia etc.) podem ser empregadas, evitando-se peso do corpo sobre o braço (exercícios de quatro apoios), movimentos rápidos e de repetição e atividades com carga e/ ou resistência.

\section{Prevenção e tratamento do linfedema do membro} superior

A prevenção do linfedema pode ser conseguida através de uma série de cuidados, que se iniciam a partir do diagnóstico de câncer da mama. Após o tratamento cirúrgico, as pacientes devem ser orientadas sobre os cuidados com a pele do membro superior homolateral ao câncer da mama, a fim de evitar possíveis traumas e ferimentos (quadro 2). O s cuidados, entretanto, devem ser repassados de forma criteriosa para que não haja um sentimento de incapacidade e impotência física. As orientações com relação à vida doméstica, profissional e de lazer devem ser direcionadas às rotinas das pacientes e condutas alternativas devem ser ensinadas quando forem realmente necessárias. 0 s exercícios devem ser iniciadosprecocemente. A auto-massagem linfática deverá ser ensinada (quadro 4), visando melhorar as vias secundárias de drenagem da linfa. Os recursos fisioterapêuticos que provocam calor superficial e profundo (ondas curtas, micro-ondas, infravermelho, turbilhão, forno de Bier, compressa quente) não devem ser utilizados no lado afetado, pois favorecem a ultrafiltração arterial para 0 interstício podendo desencadear o linfedema. ${ }^{6,11,12}$ As mulheres devem ter conhecimento sobre os sinais esintomas iniciais dos processos infecciosos e do linfedema, para que comuniquem ao médico assistente e uma correta conduta terapêutica seja implantada ${ }^{13}$. A equipe de saúde deve estar preparada para diagnosticar e intervir precocemente. 
Quadro 1. Resumos das principais complicações do câncer da mama e suas respectivas condutas

\begin{tabular}{|c|c|}
\hline \multicolumn{2}{|c|}{ Complicações cirúrgicas } \\
\hline Complicação & Conduta fisioterapêutica \\
\hline Seroma & $\begin{array}{l}\text { Exercícios com o membro superior homolateral a } 90^{\circ} \text { na flexão e abdução até a } \\
\text { alta do curativo } \\
\text { Enfaixamento torácico nos casos de seroma persistente (pouco benefício) }\end{array}$ \\
\hline Deiscência & $\begin{array}{l}\text { Exercícios com o membro superior homolateral a } 90^{\circ} \text { na flexão e abdução ou } \\
\text { sem tracionamento das bordas cirúrgicas, até a efetivação da cicatrização } \\
\text { superficial }\end{array}$ \\
\hline $\begin{array}{l}\text { Aderência, retração } \\
\text { e fibrose Cicatricial }\end{array}$ & $\begin{array}{l}\text { Manobras cicatriciais (descolamento, massagem transversa) } \\
\text { Alongamento do membro superior }\end{array}$ \\
\hline Mama fantasma & $\begin{array}{l}\text { Dessensibilização } \\
\text { Adaptação de prótese mamária } \\
\text { Recursos analgésicos (TENS / crioterapia) }\end{array}$ \\
\hline $\begin{array}{l}\text { Sintomas } \\
\text { intercostobraquiais }\end{array}$ & $\begin{array}{l}\text { Dessensibilização } \\
\text { Recursos analgésicos (TENS / crioterapia) }\end{array}$ \\
\hline Dor & $\begin{array}{l}\text { Recursos analgésicos (TENS / crioterapia) } \\
\text { Dessensibilização } \\
\text { Exercícios passivos, ativo-assistidos e ativos da cintura escapular } \\
\text { Relaxamento cervical } \\
\text { Mobilização escapular } \\
\text { Exercícios respiratórios } \\
\text { Técnicas para reeducação postural }\end{array}$ \\
\hline AIM & $\begin{array}{l}\text { Exercícios passivos, ativo-assistidos e ativos da cintura escapular } \\
\text { Técnicas de mobilização articular } \\
\text { Recursos analgésicos (TENS / crioterapia) }\end{array}$ \\
\hline Alteração postural & $\begin{array}{l}\text { Técnicas para reeducação postural } \\
\text { Adaptação de prótese mamária }\end{array}$ \\
\hline $\begin{array}{l}\text { Alterações } \\
\text { respiratórias }\end{array}$ & $\begin{array}{l}\text { Reeducação diafragmática } \\
\text { Incentivador respiratório } \\
\text { Padrão ventilatório } \\
\text { Tosse assistida }\end{array}$ \\
\hline Escápula alada & $\begin{array}{l}\text { Exercícios em posição supina para estabilização da escápula } \\
\text { Exercícios passivos, ativo -assistidos e ativos da cintura escapular, com ou sem } \\
\text { estabilização da escápula (bandagens e/ou órteses) } \\
\text { Eletroestimulação } \\
\text { Técnicas de relaxamento cervical } \\
\text { Mobilização escapular }\end{array}$ \\
\hline $\begin{array}{l}\text { Trombose linfática } \\
\text { superficial } \\
\text { (fibroesclerose do } \\
\text { vaso linfático) }\end{array}$ & $\begin{array}{l}\text { Tração manual } \\
\text { Drenagem Linfática Manual (DLM) } \\
\text { Alongamento membro superior e tronco } \\
\text { Cinesioterapia ativa assistida } \\
\text { Recursos analgésicos (TENS / crioterapia) }\end{array}$ \\
\hline $\begin{array}{l}\text { Hipotrofia e fibrose } \\
\text { do músculo peitoral } \\
\text { maior }\end{array}$ & $\begin{array}{l}\text { Exercícios passivos, ativo-assistidos e ativos da cintura escapular } \\
\text { Alongamento membros superiores e tronco }\end{array}$ \\
\hline $\begin{array}{l}\text { Estiramento do plexo } \\
\text { braquial }\end{array}$ & $\begin{array}{l}\text { Exercícios passivos, ativo-assistidos e ativos da cintura escapular } \\
\text { Alongamento membros superiores e tronco } \\
\text { Dessensibilização }\end{array}$ \\
\hline $\begin{array}{l}\text { Edema na mama } \\
\text { residual }\end{array}$ & $\begin{array}{l}\text { Uso de sutiã para sustentação } \\
\text { Drenagem Linfática manual }\end{array}$ \\
\hline
\end{tabular}




\begin{tabular}{|l|l|}
\hline Complicações radioterápicas \\
\hline \multicolumn{1}{|c|}{ Complicação } & \multicolumn{1}{c|}{ Conduta fisioterapêutica } \\
\hline Fadiga & $\begin{array}{l}\text { Exercícios moderados } \\
\text { Caminhadas (20 a } 30 \text { min) }\end{array}$ \\
\hline AIM & $\begin{array}{l}\text { Exercício passivos, ativo-assistidos e ativos da cintura escapular } \\
\text { Técnicas de desbloqueio articular } \\
\text { Recursos analgésicos (TENS / crioterapia) } \\
\text { Manobras cicatriciais (descolamento, massagem transversa) }\end{array}$ \\
\hline Plexopatia & $\begin{array}{l}\text { Avaliar necessidade de estabilização do membro superior } \\
\text { Exercícios passivos, ativo-assistidos e ativos da cintura escapular } \\
\text { Recursos analgésicos (TENS / crioterapia) } \\
\text { Dessensibilização }\end{array}$ \\
\hline Complicações quimioterápicas \\
\hline Complicação & $\begin{array}{l}\text { Exercícios moderados de baixo impacto } \\
\text { Caminhadas (20 a 30 min) } \\
\text { Reeducação diafragmática } \\
\text { Técnicas de relaxamento global }\end{array}$ \\
\hline Ataxia & $\begin{array}{l}\text { Treinamento de equilíbrio e marcha } \\
\text { Técnicas de relaxamento global }\end{array}$ \\
\hline \multirow{5}{*}{ Neurotoxidade } & $\begin{array}{l}\text { Treinamento de equilíbrio e marcha } \\
\text { Técnicas de relaxamento global } \\
\text { Recursos analgésicos (TENS / crioterapia) } \\
\text { Atividade motora fina } \\
\text { Dessensibilização }\end{array}$ \\
\hline
\end{tabular}

0 tratamento do linfedema está baseado em técnicas já bem aceitas e descritas na literatura mundial, conhecida como CPT (Complex Physical Therapy), sendo que no Brasil é chamada de TFC (Tratamento Físico Complexo) ou Linfoterapia. Esse tratamento consiste da combinação de várias técnicas que atuam conjuntamente, dependendo da fase em que se encontra o linfedema, incluindo: cuidados com a pele, drenagem linfática manual (DLM), contenção na forma de enfaixamento ou por luvas/ braçadeiras e cinesioterapia específica. 0 tratamento é dividido em duas fases, sendo que na primeira o objetivo éa redução do volume do membro, tendo a duração de 2 a 6 semanas, ea segunda éa fase de manutenção e controle do linfedema. O utros tratamentos têm sido descritos na literatura, porém seus resultados não são satisfatórios quando comparados ao T FC 6, 9, 13, 14, 15.

0 tratamento fisioterapêutico do linfedema em nosso serviço é uma adaptação da TFC, devido às características da população assistida e do serviço, sendo adotadas as seguintes condutas:

- Pacientes que apresentam diferença entre os membros superiores de 2,00 a 3,00 cm em um ou mais pontos, ou diferença de 3,00 a $5,00 \mathrm{~cm}$ sem fibrose linfoestática: adaptação de malha compressiva (tamanho padrão ou sob medida), e orientação domiciliar quanto à automassagem linfática e cinesioterapia ativa. 0 retorno para reavaliação é de 30 dias com retorno para reavaliação a cada 6 meses. $\mathrm{N}$ a falha da minimização e controle do volume do membro, a paciente é encaminhada para o enfaixamento compressivo.

- Pacientes refratárias ao tratamento domiciliar, perimetria com diferença de 3,00 a 5,00 cm com fibrose linfoestática e diferença maior que $5 \mathrm{~cm}$ : 0 tratamento é realizado duas vezes por semana, por um período aproximado de 30 a 60 dias, constando de enfaixamento compressivo com ou sem dedos, auto-massagem linfática e cinesioterapia ativa. A pós redução máxima do volume do membro, é adaptada braçadeira padrão ou sob medida. As orientações domiciliares são mantidas. 0 retorno para reavaliação é realizado com 30 dias e revisto a cada 6 meses.

\section{ORIENTAÇÕES PARA A PACIENTE APÓS LINFADENECTOMIA AXILAR}

As pacientes devem ser informadas em relação aos cuidados com o membro superior homolateral à cirurgia (quadros 2 e 3). Entretanto, deve-se tomar 0 cuidado para não provocar sensação de incapacidade e impotência funcional. Elas devem ser encorajadas a retornarem as AVD's, e devem ser informadas sobre as opções para os cuidados pessoais (cutículas, depilação, etc) ${ }^{7}$. 
Q uadro 2. O rientações após linfadenectomia axilar

\section{Orientações após linfadenectomia axilar}

Mantenha a pele hidratada e limpa. Evite e trate micoses nas unhas e no braço; Evite traumatismos cutâneos (cortes, arranhões, picadas de inseto, queimaduras, retiradas de cutículas e depilação);

Use luvas de proteção ao fazer as atividades do lar (cozinhar, jardinagem, lavar louça e contato com produtos químicos);

Não use banheiras quentes e saunas;

Durante viagens aéreas, use malha compressiva;

Evite apertar o braço do lado operado (blusas com elástico; relógios, onéis e pulseiras apertadas; coleta de sangue; aferir a pressão arterial);

Fique atenta aos sinais de infecção no braço (vermelhidão, inchaço, calor local);

Evite movimentos bruscos, repetidos e de longa duração;

Evite carregar objetos pesados no lado da airurgia.

Q uadro 3. O rientação para auto-massagem linfática

Auto-Massagem Linfática
1- Movimentos circulares, com toda a mão apoiada sobre a axila do lado
oposto ao da cirurgia (aproximadamente 20 repetições).
2- Os mesmos movimentos descritos no item 1, porém na região inguinal
do mesmo lado da cirurgia (aproximadamente 20 repetições).
3- Semi-círculos, iniciando acima do local da cirurgia até a axila oposta.
Faça este trajeto 3x.
4- Semi-círculos, iniciando na axila do lado comprometido até a região
inguinal. Faça este trajeto 3x.
5- Igual ao item 2.
6- Igual ao item 1.
Repita essa massagem duas ou três vezes ao dia. Em caso de dúvidas,
consulte a equipe de Fisioterapia

Orientacões para a paciente após reconstrução mamária

As pacientes submetidas à reconstrução mamária imediata ou tardia, com o uso de extensores, prótese mamária e retalho do grande dorsal, devem ser orientadas da mesma maneira que as pacientes submetidas à linfadenectomia axilar. Q uando submetidas àreconstrução com retalho miocutâneo do reto abdominal (TRAM ), são orientadas segundo quadro 4 .

\section{ORIENTAÇ̃̃O FISIOTERAPÊUTICA PARA AS PACIENTES EM CURSO DE TRATAMENTO ADJ UVANTE}

Durante a radioterapia, devido à lesão cutânea provocada pelo tratamento, as pacientes são orientadas a usar roupas de algodão e evitar massagem no local irradiado até 0 término da irradiação. 0 s exercícios do membro superior são mantidos até a amplitude máxima tolerada pela paciente. A atividade física regular, como caminhadas, devem ser incentivadas, visando minimizar a fadiga. Em caso de linfedema prévio de membro superior ou instalado durante a vigência da radioterapia, a fisioterapia específica pode ser iniciada, respeitandose os cuidados com o local irradiado4.

$\mathrm{Na}$ vigência de tratamento sistêmico com quimioterapia e/ou hormonioterapia, as pacientes são orientadas a manter atividade física regular, exercícios ativos para relaxamento cervical e exercícios respiratórios.

\section{ORIENTAÇÃO PARA PROFISSIONAIS DE SAÚdE}

Recursos fisioterapêuticos de eletro e termoterapia que produzam calor, tais como parafina, infra-vermelho, ultra- 
som, ondas curtas, micro-ondas, forno de Bier, compressas quentes e turbilhão, devem ser evitados nas áreas de drenagem para a axila homolateral, devido ao aumento do risco de desenvolvimento de linfedema porque provocam vasodilatação local. $N$ as regiões distantes, desde que não haja neoplasia em atividade, os recursos podem ser realizados, seguindo as devidas precauções ${ }^{1,11,12}$.

As punções venosas, vacinas e injeções devem ser evitadas no membro superior homolateral, bem como aferição da pressão arterial.

\section{FISIOTERAPIA NO CUIDADO PALIATIVO}

\section{Linfedema por obstrução tumoral}

0 tratamento do linfedema ocasionado por obstrução tumoral pode ser realizado através do enfaixamento compressivo, desde que não haja trombos tumorais ou carcinomatoses difusas infiltrativas. A redução do volume é útil para melhora dos sintomas (dor, desconforto, alteração da sensibilidade, função do membro etc. $)^{15}$.

\section{Metástase óssea}

0 tratamento visa alívio da dor, prevenção de fraturas patológicas, melhora da locomoção e conseqüentemelhora da qualidade de vida. A fisioterapia deve prover estabilização das articulações acometidas, controle álgico (TEN S, mobilização passiva, técnicas de relaxamento muscular), manutenção e/ou melhora da função respiratória, otimização da independência funcional, ganho de força muscular, treinamento de marcha e equilíbrio (quando indicado). As pacientes devem ser orientadas em relação ao uso de órtese, posicionamento no leito, independência nas AVD's e exercícios domiciliares para desenvolvimento da força muscular.

\section{CONCLUSÃO}

A implantação da rotina de atendimento fisioterapêutico para pacientes submetidas a tratamento para câncer da mama tem como objetivo principal a prevenção de complicações através de condutas e orientações domiciliares, e 0 diagnóstico e intervenção precoce, visando melhorar a qualidade de vida e a redução dos custos pessoais e hospitalares. Além disso, possibilita a formação de um banco de dados contendo informações sobre fatores de risco e incidência de complicações, avaliação de condutas empregadas e 0 planejamento do serviço.

As condutas aqui apresentadas sofrem constantes modificações, de acordo com o incremento de técnicas fisioterapeuticas, disponibilidade de recursos materiais e humanos. A nossa proposta é divulgar o trabalho realizado, para que possa ser conhecido e discutido entre os outros profissionais e serviços de fisioterapia oncológica. Vale ressaltar que as condutas estão baseadas na nossa população alvo, com os recursos e condições do nosso serviço.

\section{AGRADECIMENTOS}

As autoras agradecem as funcionárias do serviço de Fisioterapia, Sra Penha Cristina M otta de M ello e Sra Zuleika Cabral da Vitória de Sousa, por tornarem possível a realização deste trabalho, pelo apoio irrestrito, pela dedicação e carinho sempre presentes. Agradecemos, também, a Sra N airzete Firmino da Silva pelo suporte ao nosso serviço, e por tornar nosso ambiente de trabalho muito acolhedor.

Q uadro 4. O rientações após reconstrução mamária (TRAM)

Orientações após reconstrução com retalho miocutâneo do reto abdominal

Decúbito dorsal com semi flexão de tronco e joelhos (15 dias);

Marcha com semi-flexão de tronco (15 dias);

Mudança de decúbito em DL contra-lateral;

Uso de malha compressiva abdominal (1 a 3 meses);

Uso de meia compressiva nas 48 horas de PO (leito);

Exercícios respiratórios;

Exercícios de MMll;

Exercícios de MS limitado a $90^{\circ}$ (até retirada dos pontos);

Contra-indicação de exercícios de fortalecimento abdominal. 


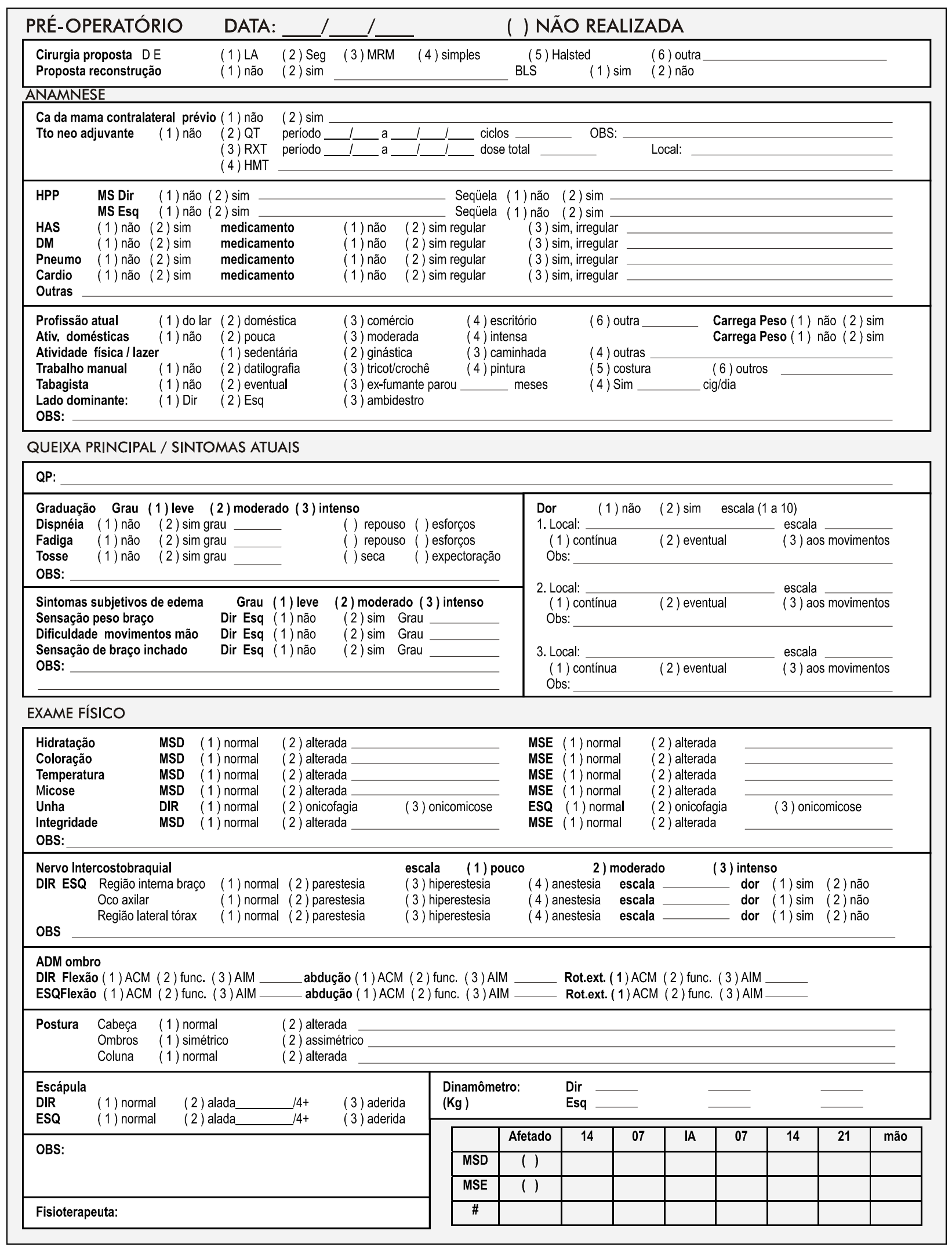

Figura 2. Avaliação fisioterapêutica pré-operatória 


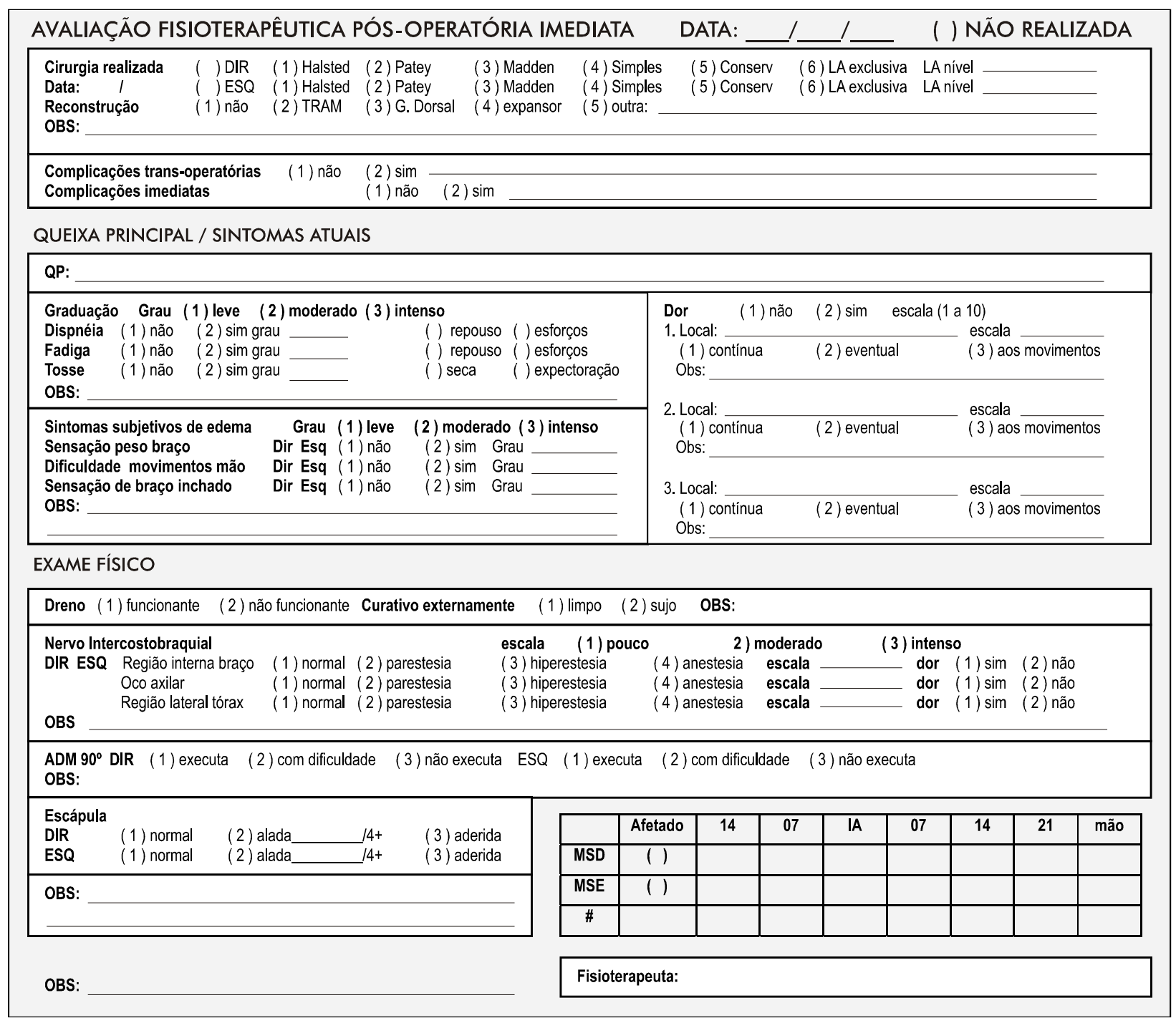

Figura 3. Avaliação fisioterapêutica pós-operatória imediata 


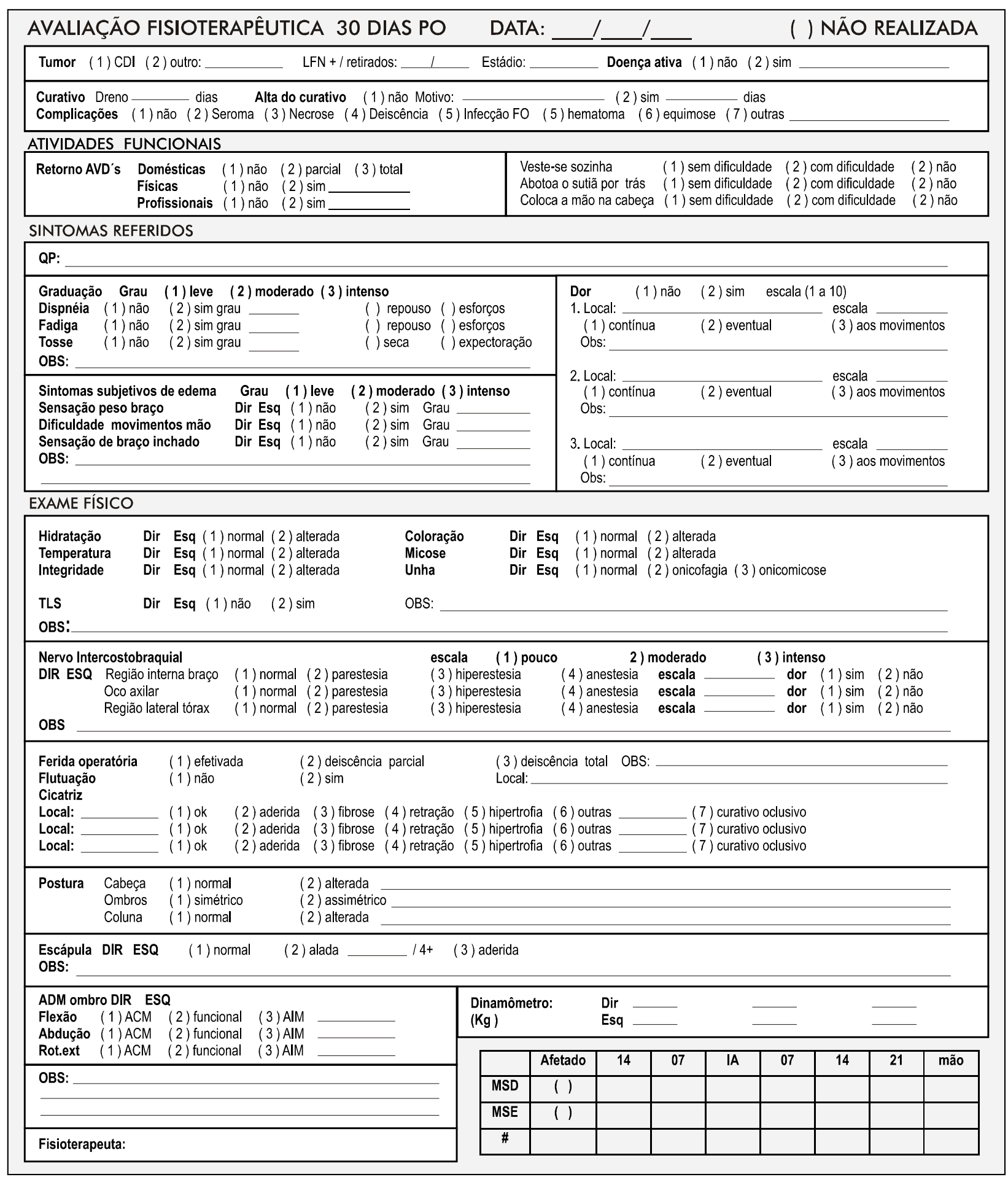

Figura 4. Avaliação fisioterapêutica pós-operatória 30 dias 


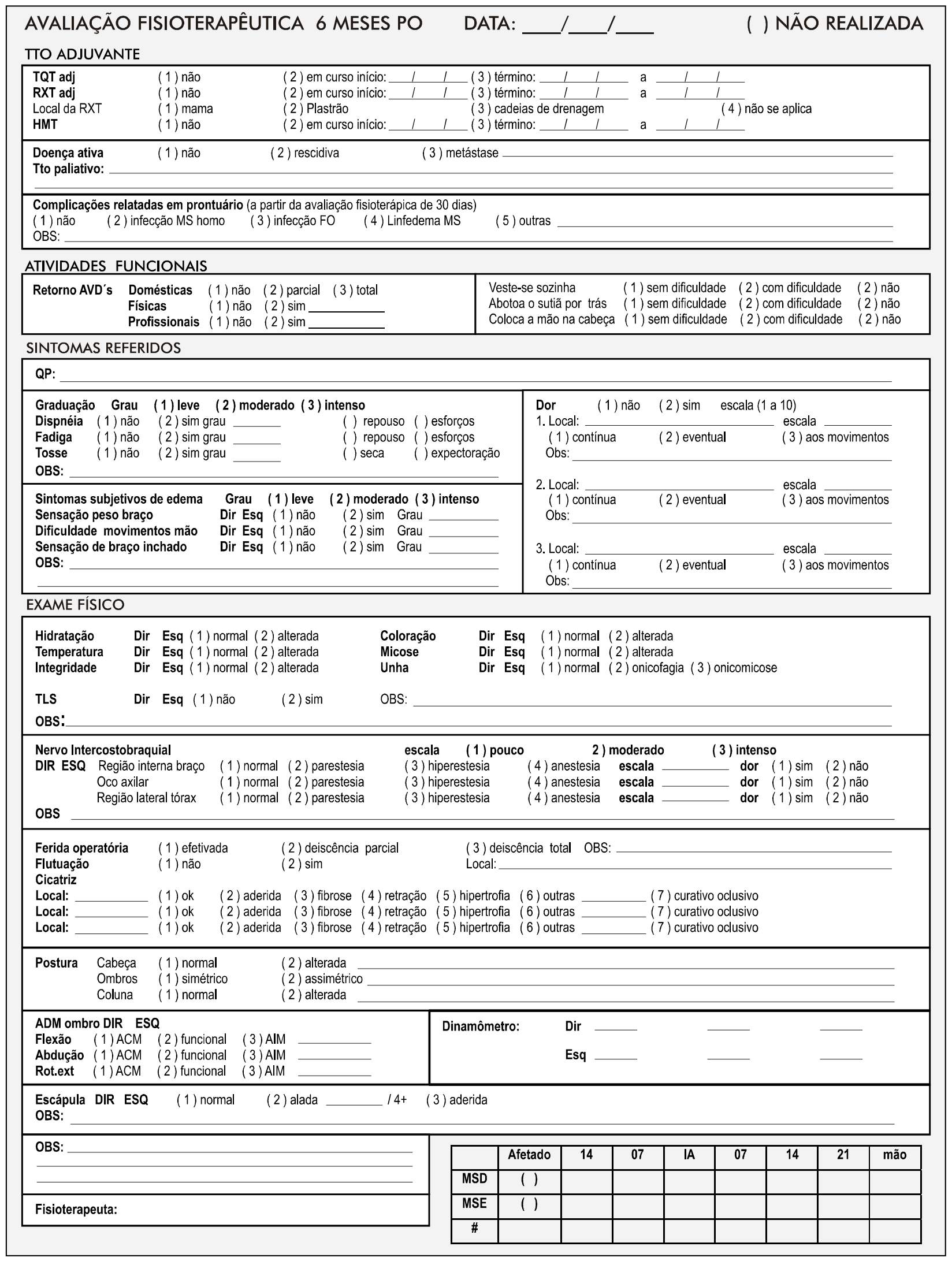

Figura 5. Avaliação fisioterapêutica pós-operatória 30 dias 


\section{BIBLO GRAFIA}

1. Bergmann A. prevalência de linfedema subseqüente ao tratamento cirúrgico do câncer demama [dissertação]. Rio deJ aneiro (RJ): EscolaN acional deSaúdePública, Fundação 0 swaldo C ruz; 2000.

2. M artino G. Prevenção e terapia das complicações. In: Veronesi U. M astologiaO ncológica. Rio deJ aneiro: Editora M edsi; 2002. p. 536-37.

3. M cAnaw M B, H arrisKW. Therole of physical therapy in therehabilitation of patients with mastectomy and breast reconstruction. Breast D is. 2002;16:163-74.

4. M ondry TE. Integration of complementary disciplinesin to theoncology clinic. Part II: Physical Therapy. Curr Probl Cancer. 2000;24(4):194-212.

5. Paci E, Cariddi A, Bianchi S, CardonaG, D istanteV, Giorgi $D$, et al. Long-term sequelæe of breast cancer surgery. Tumori. 1996; 82:321-4.

6. C amargo M , M arx AG. Reabilitação física no câncer de mama. São Paulo: Editora Roca; 2000.

7. Instituto $\mathrm{N}$ acional de Câncer; M inistério da Saúde. Controle do câncer de mama: documento de consenso. Rev Bras Cancerol. 2004;50(2):77-90.

8. Browse D, GobleD, Jones A. Axillary nodeclearance: who wantsto immobilizetheshoulder?Eur J Surg O ncol. 1996; 22:569-70.

9. Rodier J, Gadonneix P, D auplat J, Issert B, G iraud B. Influence of the timing of physiotherapy upon the lymphatic complications of axillary dissection for breast cancer. Int Surg. 1987;72(3):166-9.

10. Schultz I, Barholm M, Grondal S. D elayed shoulder exercisesin reducing seroma frequency after modified radical mastectomy: a prospective randomized study. Ann Surg O ncol. 1997;4(4):293-7.

11. Low J, Reed A. Eletroterapia explicada: princípioseprática. 3ạ ed. São Paulo: M anole; 2001.

12. Guirro E, Guirro R. Fisioterapia dermato funcional. $3 \mathrm{ed}$. São Paulo: M anole; 2004.

13. Bergmann A, M attos IE, Koifman RJ. Diagnóstico do linfedema: análise dos métodos empregados na avaliação do membro superior após linfadenectomia axilar para tratamento do câncer de mama. Rev Bras Cancerol. 2004;50(4):311-20.

14. Petrek JA, Lerner R. Linfedema. In: D oenças da mama. $\mathrm{H}$ arris J, M orrow M, 0 sborne K. 2 ed. Rio de Janeiro: MED SI; 2002.

15. Internacional Society of Lymphology. Consensus D ocument: the diagnosis and treatment of peripheral lymphedema. Lymphology. 2003;36:84-91. 